\title{
Ferromagnetic Microswimmers
}

\author{
Feodor Y. Ogrin, ${ }^{*}$ Peter G. Petrov, and C. Peter Winlove \\ School of Physics, University of Exeter, Stocker Road, Exeter EX4 4QL, United Kingdom
}

(Received 9 August 2007; published 28 May 2008)

\begin{abstract}
We propose a model for a novel artificial low Reynolds number swimmer, based on the magnetic interactions of a pair of ferromagnetic particles: one with hard and the other with soft magnetic properties, connected by a linear spring. Using a computational model, we analyze the behavior of the system and demonstrate that for realistic values of the parameters involved, the swimmer is capable of self-propelling with average speeds of the order of hundreds of micrometers per second.
\end{abstract}

PACS numbers: 47.63.mf, 45.40.Ln, 87.19.rs, 87.19.ru

The past few decades have seen increased interest in low Reynolds number swimming mechanisms with research along two complementary lines. First, the need to understand how micrometer-sized organisms are able to propel themselves in a world with no inertia has led to important insights, for example, the discovery and analysis of the rotating bacterial flagella [1-4]. The second line is more utilitarian and aimed at the construction of autonomous microrobots, capable of performing useful functions such as active targeted drug delivery, destroying kidney stones [5], or stirring and pumping in microfluidic devices [6]. Every low Reynolds number swimmer, natural or artificial, however, must fulfil two requirements. The first arrives from the fact that for micrometer-sized swimmers in aqueous environments the fluid flow is dominated by viscous effects. This situation is best illustrated by the "scallop theorem" [7]: the trajectory of the swimmer is entirely determined by the sequence of geometrical configurations it assumes and self-propulsion is only possible if the forward- and backward-motion phases in a full swimming cycle are nonreciprocal. As a result, a great deal of effort has been invested in devising various shape sequences that would lead to translational motion [8-11] and analyzing the efficiency of swimming [12-15]. However, this is only part of the problem. The second essential requirement for a successful swimmer is an actuation mechanism generating the necessary shape sequence and the requisite energy. This question remains largely unresolved, with a few proposed mechanisms holding promise, such as mechanochemical coupling in elastic membranes $[16,17]$ or the use of magnetic torque [18] or electric fields [6,19]. Plagiarizing from nature is of little help: the flagellar rotor, for example, is an extremely sophisticated mechanism, consisting of over 20 components packed in a tiny volume [20], which is impossible to reproduce with current technology. The first artificial microswimmer with a flexible flagellumlike tail has recently been assembled [21]. However, this is still a complicated assembly of magnetic particles bridged by DNA molecules and attached to a red blood cell, which makes its routine production untenable.

In this Letter we propose a model of the first ferromagnetic swimmer, a magnetically driven device, which will generate linear motion by using nonreciprocal displacement of its components. The design is simple, consisting of three elements only, and in contrast to previous models, e.g., $[10,11]$, the displacement of the components of the swimmer is not imposed but is the result of physical interactions between them. Thus, swimming is derived from a realistic actuation mechanism, which, together with the swimmer's simple design and the relatively high displacement velocities achieved, makes it an excellent candidate for experimental implementation. Compared to previous methods [21], in which the driving magnetic forces originate from the torque exerted by the external field, in our model the swimmer utilizes two types of interactions, the dipolar gradient forces between the magnetic particles and the torque exerted by the external field. The basis of the approach is to employ a pair of ferromagnetic dipoles (subsequently referred as the "dipole pair"), one with hard and the other with soft magnetic properties. The particles are sufficiently close as to interact with each other, and in many applications they will be bound to an elastic element such as a vesicle, a polymer or a protein fiber. The elastic element serves a dual purpose. First, it acts as a coupling to maintain an average separation between the particles, and second, it may constitute the "transported" object in applications aimed at targeted delivery (e.g., a giant vesicle loaded with an active agent). The dipole pair is subjected to an alternating uniform external magnetic field, allowing a hysteresis-dependent orientation of the average magnetic moments. The amplitude of the external field is chosen to be sufficient to switch the orientation of the soft particle, but lower than the switching field of the hard particle (see Fig. 1). Thus the two particles will respond differently to changing the direction of the external field. In general, the soft particle will always direct its moment along the field, whereas the hard particle will keep the moment close to the initial position of equilibrium (i.e., the local energy minimum). As demonstrated in Fig. 1, in a simplified case when the external field axis coincides with the line connecting the two dipoles, and is parallel to the anisotropy directions of the particles, the alternating magnetic field will produce alternately changing configurations with either parallel or 

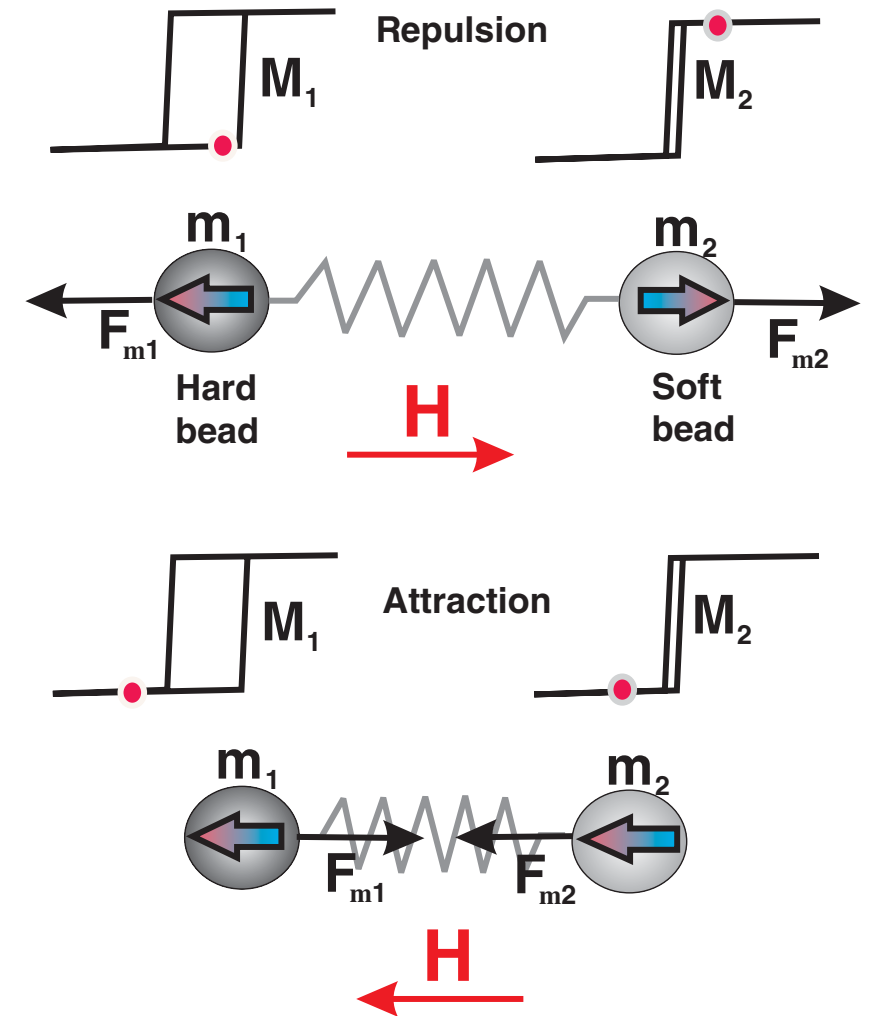

FIG. 1 (color online). Diagrammatic representation of the system consisting of a hard and a soft magnetic particle connected by an elastic element. The magnetic forces experienced by the particles are shown for two orientations of the external magnetic field $\mathbf{H}$.

antiparallel orientation of the magnetic moments. Consequently, due to the local gradient of the magnetic fields produced by the moments, the particles will be subjected to an alternating force of attraction and repulsion, leading to translational displacements along the line connecting the particles. The motion of the particles is in this case reciprocal, since the system possesses only a single degree of freedom, so the dipole pair oscillates around a fixed center. As demonstrated by the scallop theorem, in order to achieve translation of the center of mass the system has to undergo nonreciprocal cyclic displacements in its configurational space [7]. One possible realization presented here is to introduce an elliptically rotating uniform magnetic field in the $x y$ plane, $H_{x}=$ $H_{x}^{0} \cos (\omega t)$ and $H_{y}=H_{y}^{0} \sin (\omega t)$, where $\omega$ and $t$ represent the angular frequency and time, respectively, and $H_{x}^{0}$ and $H_{y}^{0}$ are the horizontal and vertical amplitudes of the field $\left(H_{y}^{0}<H_{x}^{0}\right)$. In this case the external field vector is rotated in the plane of the two dipoles. The magnetization of the soft particle follows the field direction, whereas the magnetization of the hard particle only "rocks" around an established average orientation. As a consequence, the particles are again repelled and attracted, but the instantaneous displacements of the particles are more complex leading to a non linear trajectory of motion.
To investigate the plausibility of such a system we have developed a computational model, which calculates the trajectories of the two particles under conditions of environment and geometry that provide a realistic representation of possible microscopic swimmers. The model assumes that both magnetic beads are "point dipoles" and thus will generate a dipole magnetic field, the gradient of which will result in a force of attraction or repulsion between the beads:

$$
\mathbf{F}_{M j}=\nabla\left(\mathbf{m}_{j} \cdot \mathbf{B}_{k}\right), \quad \mathbf{B}_{k}=\frac{\mu_{o}}{4 \pi}\left(\frac{3\left(\mathbf{m}_{k} \cdot \mathbf{r}_{k}\right) \mathbf{r}_{k}}{r_{k}^{5}}-\frac{\mathbf{m}_{k}}{r_{k}^{3}}\right),
$$

where $\mathbf{B}_{k}$ is the flux density generated by the bead $k, \mathbf{m}_{k}=$ $\mathbf{M}_{k} V_{k}$, and $\mathbf{M}_{k}, V_{k}$, and $\mathbf{r}_{k}$ are the magnetization, volume, and position vector of the beads $j$ and $k$, respectively (indices denote the particle number, $j=1$ or $j=2$ and $k=3-j$; see Fig. 1). To determine the orientation of the dipole moments in the external field $\mathbf{H}$ the model uses a standard magnetostatic approach [22], in which the interaction energy is minimized to find the local minima with stable positions of both moments. For simplicity, the magnetostatic energy density for each particle is represented by two terms, $E=-\mathbf{M} \cdot \mathbf{H}+K \sin ^{2} \alpha$. The first term is the Zeeman energy representing the interaction of the magnetic moments with the applied field $\mathbf{H}$, and the second, the uniaxial magnetic anisotropy term, describes the combined effects of shape and/or crystalline anisotropy. In the latter, $\alpha$ is the angle between the magnetic moment of the particle and the orientation of its easy axis with respect to the vector $\mathbf{M}$, and $K$ is the effective anisotropy field constant. For the hard particle $K$ is chosen so that the effective anisotropy field is higher than the value of the applied field $(2 K / M>H)$, whereas the soft particle has a zero value of $K$. It is assumed that the time scale of magnetic switching/ relaxation $(<1 \mathrm{~ns})$ is significantly shorter than the time period of the applied field oscillation $(\approx 1-10 \mathrm{~ms})$. It should be noted that the choice of different anisotropy constants for the beads is important not only for obtaining different switching characteristics, but also for generating asymmetric torques. Under the action of magnetic torque, $\mathbf{T}=\mu_{0} \mathbf{m} \times \mathbf{H}$, the particle with higher anisotropy can rotate to align its easy axis with the direction of the applied field. If the particles are rigidly connected with each other, the torque applied to the hard particle will lead to rotation of the whole dipole pair, which will provide another degree of freedom in the configurational space. The soft particle does not contribute to the torque. Its anisotropy is negligible, so the rotation of its magnetic moment does not affect the orientation of the pair.

As well as the magnetic interaction, both beads are subjected to elastic forces and hydrodynamic friction. The elastic force $\mathbf{F}_{E}$ results from the extension or compression of the elastic element connecting the two particles, $\mathbf{F}_{E}=k_{s}\left[\left(\mathbf{r}_{2}-\mathbf{r}_{1}\right)-\mathbf{r}_{0}\right]$. Here $k_{s}$ is an effective spring constant, and $\mathbf{r}_{0}$ is the vector representing a relaxed spring. The bending modulus of the spring is assumed to be 
infinitely large. The viscous resistance on each particle is calculated using the Stokes approximation, in which the drag force $\mathbf{F}_{D}$ is proportional to the particle velocity $\boldsymbol{v}$, radius $R$, and the viscosity of the fluid $\eta, \mathbf{F}_{D}=-6 \pi \eta R \boldsymbol{v}$.

The motion of the system has been calculated for realistic parameters of the liquid (water, $\eta=10^{-3} \mathrm{Pas}$ ) and the dipole-pair system (magnetic particles of 3.2 and $1.6 \mu \mathrm{m}$ diameter connected by a $20 \mu \mathrm{m}$ long elastic element with $k_{s}=3 \times 10^{-3} \mathrm{~N} \mathrm{~m}^{-1}$ ). Figure 2 shows the trajectories of the particles and their center of mass. As well as the motion of the individual particles, there is a linear displacement, or "swimming," of their center of mass, with an average speed shown in inset (i). Inset (ii) shows the trajectory in the configurational space of the systems with coordinates $l$ (the distance between the centers of the two magnetic particles) and $\theta$ (the angle of the bond between them with respect to the horizontal axis) forming a closed loop. After a full cycle the original configuration of the system is recovered, but the shape sequence executed is nonreciprocal; thus, the conditions for swimming are fulfilled allowing a net translation of the center of mass.

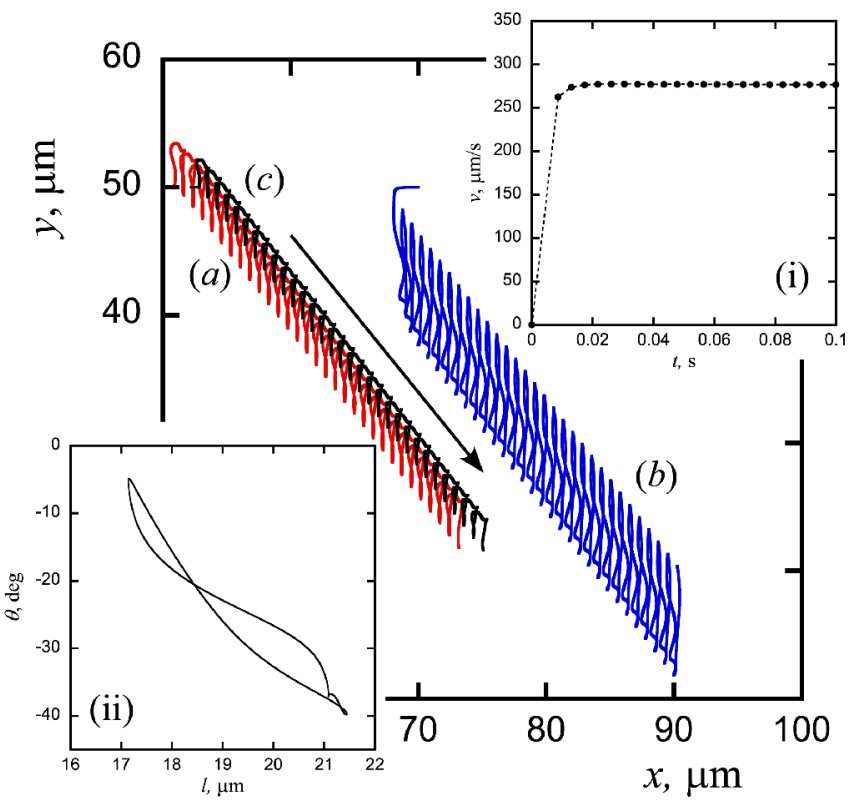

FIG. 2 (color online). Trajectories of the hard bead [(a), red], the soft bead [(b), blue], and their center of mass [(c), black], after application of an elliptically rotating external field. As well as rapid oscillatory motion, the dipole pair follows a directional linear displacement ("swimming") along the arrow towards the lower-right corner [24]. Inset (i) shows the mean speed of the center of mass as a function of time. Inset (ii) shows the trajectory of the swimmer in its configurational space where $\theta$ is the angle between the elastic element and the horizontal axis and $l$ is its instantaneous length. The parameters of the simulation are $H_{y}^{0}=1 \mathrm{kOe}, H_{x}^{0}=5 \mathrm{kOe}, f=230 \mathrm{~Hz}, 2 K_{1} / M_{1}=$ $10 \mathrm{kG}, 2 K_{2} / M_{2}=0 \mathrm{kG}, M_{1}=M_{2}=1.4 \times 10^{6} \mathrm{~A} \mathrm{~m}^{-1}, 2 R_{1}=$ $3.2 \mu \mathrm{m}, 2 R_{2}=1.6 \mu \mathrm{m}, r_{0}($ relaxed spring length $)=20 \mu \mathrm{m}$, $k_{s}=3.0 \times 10^{-3} \mathrm{~N} \mathrm{~m}^{-1}, \eta=10^{-3} \mathrm{Pas}, \mathrm{Re} \approx 10^{-3}$.
Figure 3 demonstrates the different phases over one cycle together with the corresponding orientation of the magnetic moments and the magnetic dipolar gradient forces. The trajectory of motion and thus the characteristics of swimming of the dipole pair depend on the parameters of both the particles and the external environment. Linear motion requires first that the particles must have different size, and second, that the time variation of the external field must not lead to full rotation of the hard bead. The latter requirement can be achieved by choosing an appropriate value for $H_{y}^{0}$. It is interesting to note the significance of the torque in this system. On the one hand, it is essential for overall displacement of the dipole pair. Without the torque, even if the external field is rotated, there is no displacement of the center of mass. In this case the particles oscillate in phase with the field, but the center of mass is either stationary, if the particles are of the same size, or oscillating without translation. Generating a large torque, on the other hand, leads to a circular rotation (instead of "rocking") of the hard particle, thus also preventing linear motion.

Figure 4 demonstrates the dependence of the linear translational speed of the dipole pair on the magnetization $M$ and the spring coefficient $k_{s}$ (both represented as dimensionless ratios against the viscous drag). The speed is weakly dependent on $k_{s}$, but varies considerably with the magnetization. The swimmer's speed also depends on other external parameters such as the driving frequency $f$ and the viscosity of the environment $\eta$ (data not shown), which provides flexibility in controlling the swimming behavior. Compared with the average velocities produced by micron-sized organisms such as E. coli ( $\approx 30 \mu \mathrm{m} \mathrm{s}^{-1}$ $[20,23])$, the speed of the dipole pair can be up to an order of magnitude higher (see Fig. 4, right-hand axis). The

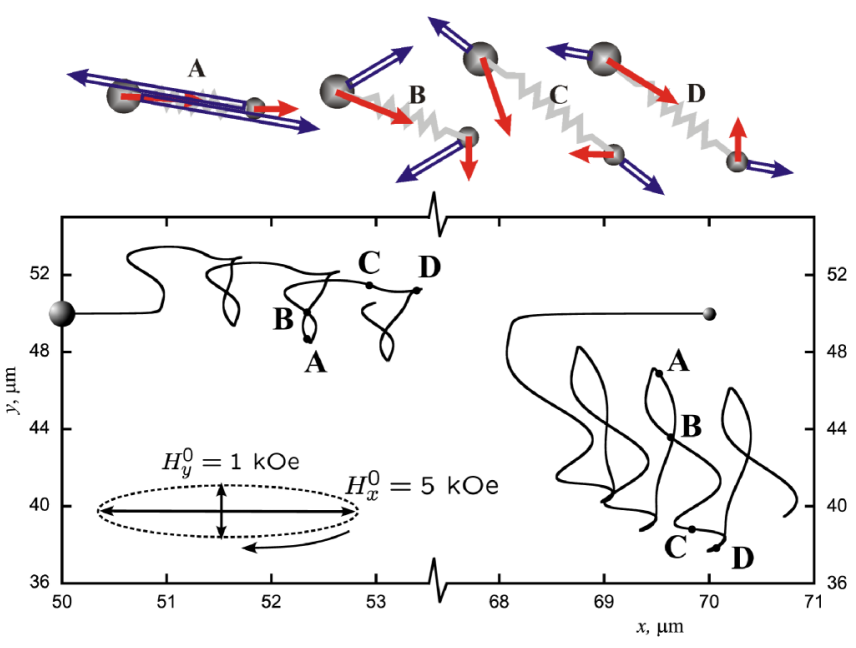

FIG. 3 (color online). Particle trajectories (solid lines) at different phases of the field cycle. The diagram shows the orientations of the magnetization for each particle (top single arrows), the magnetic gradient forces (double-line arrows), and the orientation of the dipole pair at the same points of the cycle. The anisotropy easy axis of the hard particle coincides with the line connecting the particles. 


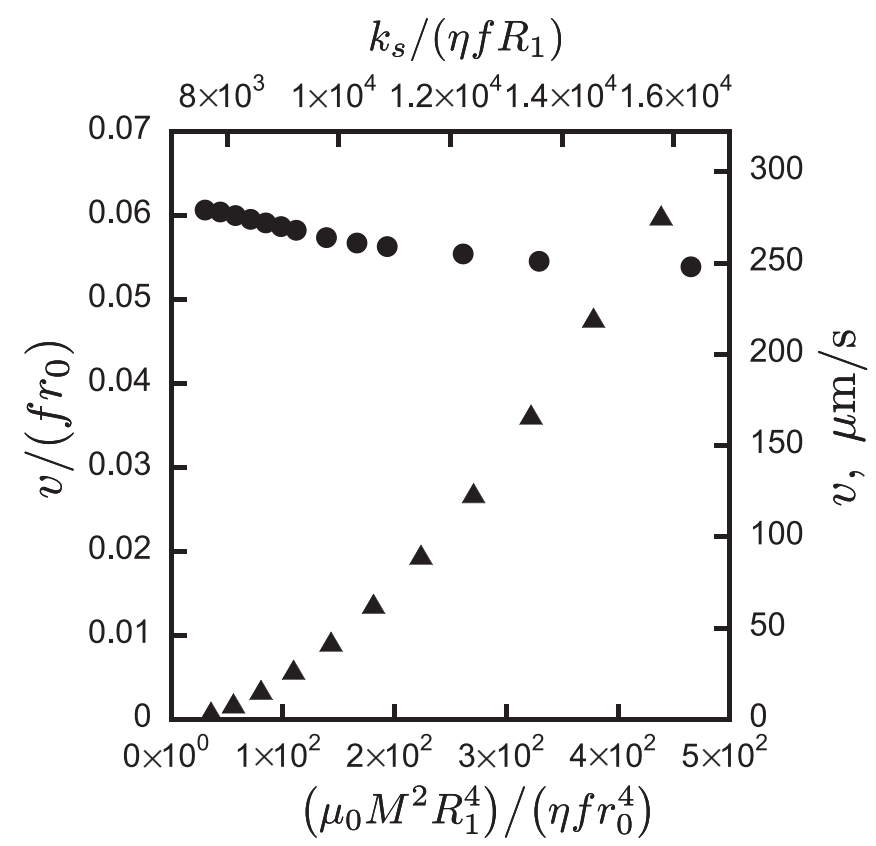

FIG. 4. Dimensionless speed of translational displacement of the swimmer, $v /\left(f r_{0}\right)$, as a function of the spring constant $k_{s}$ (circles) and magnetization $M_{1}=M_{2}=M$ (triangles), both scaled against the viscous drag. The values of the other parameters are as in Fig. 2. The right-hand axis shows the absolute speeds attained by the swimmer.

dimensionless speed, $v /\left(f r_{0}\right)$, is a direct measure of the swimming efficiency and compares the average distance travelled per cycle to the size of the swimmer, $r_{0}$. For experimentally realistic values of the parameters, a single cycle may lead to a displacement comparable to the particle size, $R_{1}$.

The model demonstrates the viability of a microscopic swimmer driven by magnetic dipole interactions, even though a number of simplifications have been made. The elastic element is exaggerated in length to hydrodynamically decouple the two magnetic particles. Its bending is not included and its detailed geometry disregarded. Shorter elastic couplings, for example, would exhibit swimming albeit with modified dynamics due to the hydrodynamic coupling and a contribution of the spring motion to the viscous drag. The particular geometry of the swimmer, along with the exact elastic behavior of the spring (including stretching and bending), could be treated numerically. Such refinements, however, although important for a practical swimmer, would add little to the proof of concept reported in this Letter.

By balancing the parameters of the system it is possible to optimize the swimming characteristics. A simplification has been made in setting $K=0$ for the soft particle. In real materials $K$ will always be nonzero, so the soft particle will also rotate in the field. This may counteract the torque produced by the hard particle, but the anisotropy of the hard particle can always be chosen larger than that of the soft particle, to ensure that the torque produced by the hard particle is larger. The torque can also be increased by making the volume of the hard particle larger.

Based on the above theoretical principles we have constructed a macroscopic prototype of the swimmer [24]. The video available in [24] demonstrates the swimming of the device in the glycerol-air interface (estimated $\mathrm{Re} \approx 10^{-5}$ ) under different conditions of the external field amplitude and frequency. The detailed study of the experimental prototype will be presented elsewhere.

The authors acknowledge the contribution of Misha Portnoi in providing useful comments and suggestions.

*F.Y.Ogrin@exeter.ac.uk

[1] H. C. Berg and R. A. Anderson, Nature (London) 245, 380 (1973).

[2] M. Silverman and M. Simon, Nature (London) 249, 73 (1974).

[3] S. H. Larsen et al., Nature (London) 249, 74 (1974).

[4] H. C. Berg, Nature (London) 249, 77 (1974).

[5] J. Edd et al., in Proceedings of the IEEE/RSJ International Conference on Intelligent Robots and Systems (IEEE, New York, 2003), Vol. 3, pp. 2583-2588.

[6] S. T. Chang et al., Nat. Mater. 6, 235 (2007).

[7] E. M. Purcell, Am. J. Phys. 45, 3 (1977).

[8] S. Camalet et al., Phys. Rev. Lett. 82, 1590 (1999).

[9] L. E. Becker et al., J. Fluid Mech. 490, 15 (2003).

[10] A. Najafi and R. Golestanian, Phys. Rev. E 69, 062901 (2004).

[11] J. E. Avron et al., New J. Phys. 7, 234 (2005).

[12] A. Shapere and F. Wilczek, J. Fluid Mech. 198, 557 (1989); 198, 587 (1989).

[13] E. M. Purcell, Proc. Natl. Acad. Sci. U.S.A. 94, 11307 (1997).

[14] J. E. Avron et al., Phys. Rev. Lett. 93, 186001 (2004).

[15] D. Tam and A. E. Hosoi, Phys. Rev. Lett. 98, 068105 (2007).

[16] R. Lipowsky, Statistical Mechanics of Biocomplexity, Lecture Notes in Phys. Vol. 527 (Springer, Berlin, 1999), pp. 1-23.

[17] P. G. Petrov et al., Europhys. Lett. 48, 435 (1999).

[18] K. Ishiyama et al., Sens. Actuators A, Phys. 91, 141 (2001).

[19] Y. Osada et al., Nature (London) 355, 242 (1992).

[20] H. C. Berg, Proc. Natl. Acad. Sci. U.S.A. 93, 14225 (1996).

[21] R. Dreyfus et al., Nature (London) 437, 862 (2005).

[22] G. Bertotti, Hysteresis in Magnetism (Academic, San Diego, 1998).

[23] W. R. DiLuzio et al., Nature (London) 435, 1271 (2005).

[24] See EPAPS Document No. E-PRLTAO-100-046818 for an animation of the swimmer and video of the constructed microscopic prototype. For more information on EPAPS, see http://www.aip.org/pubservs/epaps.html. 